\title{
Spectral mapping of natural signals
}

\author{
Md. Shoaibur Rahman \\ Baylor College of Medicine, Houston, TX 77030 \\ Current address: Shell Technology Center, Houston, TX 77082 \\ shoaibur.rahman@ $\{$ bcm.edu, shell.com $\}$
}

\begin{abstract}
Here we present an algorithm to procedurally map spectral contents of natural signals. The algorithm takes in two inputs: a signal whose spectral component needs to be mapped and a warping or mapping function. The algorithm generates one output, which is a mapped version of the original signal. The input signal is mapped into the output signal in two steps. In the analysis step, the algorithm performs a series of operations to modify the spectral content, i.e., compute the warped phase of the signal according to the given mapping function. In the synthesis step, the modified spectral content is combined with the envelope information of the input signal to reconstruct the warped or mapped output signal.
\end{abstract}

\section{Introduction}

This paper presents an algorithm that procedurally maps spectral contents of natural signals to generate a modified and desired output signal. The spectral mapping of natural signals yields numerous applications, including in music and acoustic engineering, language processing, and the development of various artificial intelligence technologies. For example, computer music composers are interested in pitch transposition by modifying spectra of a wide variety of sound signals. Systematic pitch transpositions could produce many of our desired perceptual effects of sound signals. To achieve these perceptual effects, efficient mapping of spectral contents of sound signals has been a great importance in audio applications for a long time (Grey and Moorer, 1977; Grey and Gordon, 1978; Samson et al., 1997; 
Krumhansl and Iverson, 1992; Alluri and Toiviainen, 2010; Vos and Rasch, 1881; Deutsch, 2013). The importance of spectral mapping has been widely implied in other analogous fields as well, e.g., in acoustic oceanographic measurements (Niu et al., 2014; Bonnel et al., 2017), and in clinical applications using EEG (Chemin et al., 2018; Wang et al., 2016) and fMRI (Meszlényi et al., 2017). The spectral mapping of these signals allows us to better understand the important features and patterns in the signals. Another emerging field of applications of spectral mapping is the artificial intelligence. Recent studies reported and implied the significance of spectral mapping in natural language processing, e.g., speech recognition (Chang et al., 2017; Goupell et al., 2008; Noda, 1988; Lee and Rose, 1998), speech enhancement and reverberation (Han et al., 2015), and also in computer vision, e.g., mapping of image features, image debluring and image segmentation (Qiang et al., 1996).

The process of spectral mapping is equivalent to the warping of each frequency in the signal. Therefore, the mapping can be achieved by warping the instantaneous frequencies of the input signals based on a given mapping function (Fig. 1). The idea of frequency warping was initially proposed in terms of phase vocoders (Flanagan and Golden, 1966; Moorer, 1978), which was originally developed for timescale modification of audio signals and were implemented using filter-banks and Fourier transforms (Dolson, 1986). Then the improved versions of the phase vocoders were reported over the time (Puckette, 1995; Laroche and Dolson, 1999). However, although the implementation techniques of the later versions were different from the initial ones, the goal was the same - to change the temporal characteristics of a sound by stretching or compressing the time-base of the spectrogram. The main disadvantage of these methods is that they introduce errors in the spectral mapping process (Puckette and Brown, 1998). Therefore, further efforts had been made to develop more general methods for frequency warping, for instance, using arbitrary allpass maps (von Schroeter, 1999), and more recently, using short time Fourier transforms and Gabor frames (Evangelista and Cavaliere, 2007; Mejstrik and Evangelista, 2016; Wabnik et al., 2005). These methods perform well for mapping spectral contents of simple signals like pure tones or even frequency sweeps. However, these algorithms are susceptible to high spectral leakage (error) during the 
mapping process for complex natural signals like human speech and multi-channel acoustic signals.

To address this gap, here we present an algorithm that maps spectral contents of an input signal to a desired output signal. Several experiments were performed to validate the algorithm. The validity of the mapped signals was tested using signals of different complexity levels, e.g., from pure tones to human voice with instruments. The experiment results show that although the algorithm concedes a nominal spectral leakage, it can faithfully map the spectra of the signals to the desired ones.

\section{Algorithm}

The proposed algorithm for spectral mapping of natural signals has been developed using fundamental signal processing and transformation theorems (Rahman, 2019). A high-level description of the algorithm is presented in a flow diagram (Fig. 2), and is summarized in a table (Table 1). The algorithm takes an input signal and processes it in two steps: analysis and synthesis steps. The analysis step comprises the Hilbert transformation that computes an envelope (i.e., smooth outlines of the extremes of the signals) and the phase information of the input signal. The extracted phases are mapped or warped using the given mapping or warping function. The synthesis step, on the other hand, combines the envelope information and the warped phases to reconstruct an output signal, which is a mapped or warped version of the input signal. The details of the inputs, the analysis and synthesis steps, and the outputs are explained in the following subsections.

\subsection{Inputs}

The algorithm requires two inputs: (1) A real signal $x[n]$ in the time domain with a length of $M \in \mathbb{Z}_{+}$with $\mathrm{n}$ being the time index; (2) A frequency mapping or warping function, i.e., a function that explains the relationship between the instantaneous frequencies of the input signal and the desired output signal at each time point. We denote the mapping function as $\tilde{f}[\mathrm{n}]=$ func $(\mathrm{f}[\mathrm{n}])$, where $\mathrm{f}$ denotes frequency, and 
$\mathrm{f}[\mathrm{n}]$ and $\tilde{\mathrm{f}}[\mathrm{n}]$ are the frequencies of the input and output signals at time $\mathrm{n}$, respectively.

\subsection{Analysis step}

2.2.1. Construct analytic signal: An analytic signal constructed using four computational steps (Eqn. 1a-d). First, a mean signal $\mu$ is computed from the input signal (Eqn. 1a). Second, an operation signal s[n] is computed by subtracting the mean signal from the input signal (Eqn. 1b). Third, the Hilbert transform is applied on the operation signal (Eqn. 1c). Forth, the operation signal and Hilbert transform of the operation signal are used to form the analytical signal $\mathrm{s}_{\mathrm{a}}[\mathrm{n}]$, which is a complex signal in time domain the real and imaginary parts of the analytic signal is the operation signal and the Hilbert transform of the operation signal, respectively. (Eqn. 1d). The analytic signal does not contain any negative frequency, unlike Fourier transforms; therefore, it makes the computation for reconstruction easier.

$$
\begin{gathered}
\mu=\frac{1}{M} \sum_{k=1}^{M} x[k] \\
s[n]=x[n]-\mu \\
\widehat{s}[n]=\mathcal{H}(s[n]) \\
s_{a}[n]=s[n]+j \hat{s}[n]
\end{gathered}
$$

where, $\mu, s[n], \hat{s}[n]$ and $s_{a}[n]$ denote the mean signal, operation signal, Hilbert transform of the operation signal, and the analytic signal, respectively. $\mathcal{H}$ represents an operator for Hilbert transform.

2.2.2. Extract properties of the analytic signal: The analytic signal constructed in the previous step 
contains two fundamental properties, which can be extracted by simple algebraic operations (Eqn. 2a-b). The first property is the envelope e[n] that stores the information about how the amplitude of the signal changes in time, and is computed as the magnitude of the analytic signal (Eqn. 2a). The second property is the instantaneous phase $\phi[\mathrm{n}]$ that stores the information about the location of a point on the signal waveform at any given time, and is computed as the angle of analytic signal (Eqn. 2b).

$$
\begin{gathered}
\mathrm{e}[\mathrm{n}]=\sqrt{|\mathrm{s}[\mathrm{n}]|^{2}+|\hat{s}[\mathrm{n}]|^{2}} \\
\phi[\mathrm{n}]=\arctan \left(\frac{\hat{s}[\mathrm{n}]}{\mathrm{s}[\mathrm{n}]}\right)
\end{gathered}
$$

The phases are represented in radians. In the subsequent operations in the analysis step, only phase information is used, i.e., the phases are mapped or warped based on the given mapping or warping function. The envelope information remains intact, and is used only for reconstructions during the synthesis step.

2.2.3. Compute warped phases: The instantaneous phases are converted into new phases, which are also known as mapped or warped phases. This conversion process is performed using a 5-step procedure: two forward steps that involve phase unwrapping and differential operations, one mapping step that involves frequency mapping, and two backward steps that involves integral and phase wrapping operations (Shoaib et al. 2010; Rahman, 2011; Rahman and Haque 2012; Rahman, 2019).

Forward step 1 - phase unwrapping: The computed phases (using Eqn. 2b) are unwrapped to obtain the actual phases of the input signal. The computed phases of the signal (using Eqn. $2 b$ ) lies between $-\pi$ and $\pi$, which may not be the actual phase of the signal. For instance, let's consider two sinusoids: $\sin (2 \pi+$ $\pi / 3)$ and $\sin (-4 \pi+\pi / 4)$. The actual phases of these two signals are $2 \pi+\pi / 3$ and $-4 \pi+\pi / 4$, 
respectively. However, the computed phases (using Eqn. $2 b$ ) of the signals are $\pi / 3$ and $\pi / 4$, respectively. Therefore, we need to restore the actual phases by adding appropriate multiples of $2 \pi$ to the phases computed (using Eqn. 2b). This process of restoring the actual phases from the computed phases is called phase unwrapping. Mathematically, the unwrapped phases are computed as (Eqn. 3a):

$$
\theta[\mathrm{n}]=\mathfrak{U}(\phi[\mathrm{n}])
$$

where, $\theta[\mathrm{n}]$ denote the unwrapped or actual instantaneous phases and $\mathfrak{U}$ denotes the unwrapping operator.

Forward step 2 - phase to frequency conversion using differential operation: The actual instantaneous phases are converted into instantaneous frequencies. This conversion is required because the given mapping or warping function contains the relationship between the frequencies (not phases) of the input and the desired output signals. We compute instantaneous frequencies from the actual instantaneous phases by applying a differential operation on the actual instantaneous phases (Eqn. 3b), i.e.,

$$
f[n]=\frac{F_{s}}{2 \pi}(\theta[n+1]-\theta[n])
$$

where, $f[n]$ is the instantaneous frequency and $F_{S}$ denotes sampling frequency.

Frequency mapping step: The instantaneous frequencies are mapped or warped based on the mapping or warping function, i.e.,

$$
\tilde{\mathrm{f}}[\mathrm{n}]=\operatorname{func}(\mathrm{f}[\mathrm{n}])
$$

Now, for the reconstruction of the output signal, we need phase information, specifically the mapped or 
warped phases, not the mapped frequencies. Therefore, after obtaining the mapped frequency, we use backward steps and apply inverse operations of the forward steps (i.e., integral and wrapping, which are opposite of the forward steps 2 and 1 , respectively).

Backward step 1 - frequency to phase conversion using integral operation: The mapped or warped frequencies, $\tilde{\mathrm{f}}[\mathrm{n}]$, are converted to mapped or warped phases $\tilde{\theta}[\mathrm{n}]$ by applying an integral operation (Eqn. 3d), i.e.,

$$
\tilde{\theta}[\mathrm{n}]=\frac{2 \pi}{\mathrm{F}_{\mathrm{s}}} \sum_{\mathrm{k}=1}^{\mathrm{n}} \tilde{\mathrm{f}}[\mathrm{k}], \quad \mathrm{n}=1,2 \ldots \mathrm{M}
$$

where, $\widetilde{\theta}[n]$ are the warped phases.

Backward step 2 - phase wrapping: The mapped or warped phases are wrapped between $-\pi$ and $\pi$. In contrast to the forward step 1 , we obtain wrapped phases by subtracting appropriate multiples of $2 \pi$ from the mapped or warped phases (Eqn. 3e), i.e.,

$$
\widetilde{\phi}[n]=\mathfrak{U}(\tilde{\theta}[n])-2 \pi\left\lfloor\frac{\mathfrak{U}(\tilde{\theta}[n])+\pi}{2 \pi}\right\rfloor
$$

where, L. $\rfloor$ denotes a floor operation and $\widetilde{\phi}[\mathrm{n}]$ denotes the instantaneous phases of the desired output signal.

\subsection{Synthesis step}

2.3.1. Reconstruct operation signal: If we obtain the operation signal for the desired output signal, then it will be easy to compute the desired output signal. Using trigonometric identity (Fig. 3B), we get that the operation signal for the desired output signal is the element-wise dot product between the envelope and 
the cosine of the phases of the desired output signal, i.e., $\tilde{s}[n]=\tilde{e}[n] \cos \widetilde{\phi}[n]$. Here, $\tilde{e}[n]$ and $\tilde{s}[n]$ are the envelope and the operation signal of the desired output signal. Noticeably, the envelope of the desired output signal is unknown; however, we want the envelope of the desired output signal to be identical to the envelope of the input signal to avoid any information losses from envelope transformation. Therefore, $\tilde{\mathrm{e}}[\mathrm{n}]=\mathrm{e}[\mathrm{n}]$, and the operation signal of the desired output signal is computed by the element-wise dot product between the envelope of the input signal and the cosine of the mapped or warped phases (Eqn. 4a), i.e.,

$$
\widetilde{s}[\mathrm{n}]=\mathrm{e}[\mathrm{n}] \cos \widetilde{\phi}[\mathrm{n}]
$$

2.3.2. Reconstruct mapped output signal: We denote the desired output signal as $\tilde{x}[n]$. The mean of $\tilde{\mathrm{x}}[\mathrm{n}]$ is $\tilde{\mu}$. So, according to the definition of the operation signal, $\tilde{\mathrm{s}}[\mathrm{n}]=\tilde{\mathrm{x}}[\mathrm{n}]-\tilde{\mu}$. The mean signal $\tilde{\mu}$ is unknown; however, a good estimation is $\tilde{\mu} \approx \mu$, which implies that $\tilde{x}[n]=\mu+\tilde{s}[n]$. Therefore, the desired output signal computed by adding the mean signal with output operation signal (Eqn. 4 b), i.e.,

$$
\tilde{\mathrm{x}}[\mathrm{n}]=\mu+\mathrm{e}[\mathrm{n}] \cos \widetilde{\phi}[\mathrm{n}]
$$

To understand how this reconstruction equation (Eqn. 4b) works at the synthesis step, here we give an example of perfect reconstruction, i.e., we set up an example so the desired output signal is identical to the input signal. An identity frequency mapping or warping function, i.e., $\tilde{\mathrm{f}}[\mathrm{n}]=\mathrm{f}[\mathrm{n}]$, is an obvious choice for the perfect reconstruction. With the identity frequency warping function, we find that the wrapped phases for original input signal and warped output signal are identical, i.e., $\widetilde{\phi}[\mathrm{n}]=\phi[n]$, after applying unwrap/wrap and differential/integral operations in the analysis steps. So, according to the reconstruction equation, the signal can be reconstructed as:

$$
\tilde{\mathrm{x}}[\mathrm{n}]=\mu+\mathrm{e}[\mathrm{n}] \cdot \cos \phi[\mathrm{n}]
$$


Using trigonometric identities (Fig. 3a):

$$
\tilde{x}[n]=\mu+e[n] \cdot \cos \arctan \left(\frac{\hat{s}[n]}{s[n]}\right)=\mu+e[n] \cdot \cos \arccos \left(\frac{s[n]}{e[n]}\right)=\mu+e[n] \cdot \frac{x[n]-\mu}{e[n]}=x[n]
$$

Therefore, with an identity frequency warping function, the warped output signal is identical to the original input signal. This verifies that equation (4b) can be used to estimate the warped output signal.

\subsection{Output}

The algorithm returns a single output, $\tilde{\mathrm{x}}[\mathrm{n}]$, which is the frequency-mapped or -warped version of the original input signal, $\mathrm{x}[\mathrm{n}]$. Since the envelope of the input signal remained intact in the analysis steps and is just used in the synthesis step, the output signal maintains the same envelope properties that were in the original input signal.

\section{Experiments - testing and validation of the algorithm}

The performance of the algorithm was validated and tested using three types of input signals: pure tone sinusoids, frequency sweep sinusoids, and natural sound signals. For simplicity and to keep the analyses consistent across the examples, same warping function was used in all examples; specifically, we used the following simple linear function for frequency mapping or warping:

$$
\tilde{\mathrm{f}}[\mathrm{n}]=2 / 3 * \mathrm{f}[\mathrm{n}]
$$

This implies the following inverse function for frequency re-mapping or re-warping:

$$
\mathrm{f}[\mathrm{n}]=3 / 2 * \tilde{\mathrm{f}}[\mathrm{n}]
$$




\subsection{Experiment 1: Validation with pure tone sinusoid}

A pure tone sinusoidal input signal was generated with a sampling frequency of $16 \mathrm{kHz}$. The frequency of the signal was $300 \mathrm{~Hz}$ (Fig. 4A). The power spectrum (PS) of the signal confirmed that its frequency was $300 \mathrm{~Hz}$, i.e., the power of the signal was concentrated around $300 \mathrm{~Hz}$ (Fig. 4B). The sinusoidal signal and the mapping function were given as the inputs to the algorithm, which outputted another sinusoidal signal but with a different frequency (Fig. 4C). Theoretically, according to the mapping function (Eqn. 5a), the frequency of the mapped output signal should be $200 \mathrm{~Hz}$, i.e., $\tilde{\mathrm{f}}=2 / 3 * 300=200 \mathrm{~Hz}$. The power spectrum confirmed that the frequency of the mapped output signal was $200 \mathrm{~Hz}$ (Fig. 4D).

Now, we would like to investigate whether this input-output mapping is lossless. To test this, we used the mapped output and the inverse re-mapping function as the inputs to the algorithm, i.e., the frequency of the input signal is $200 \mathrm{~Hz}$ and the mapping is equivalent to the re-mapping function (Eqn. $5 \mathrm{~b}$ ).

Theoretically, the algorithm should produce an output that is identical to the original input signal, i.e., a sinusoid with a frequency of $\mathrm{f}=3 / 2 * 200=300 \mathrm{~Hz}$. We computed the re-mapped output signal (Fig. 4E) and compared that with the original input signal in two ways. First, we computed the cross-correlation $(\mathrm{r})$ between this re-mapped and the original signals, which was unity, i.e., $\mathrm{r} \approx 1$. Second, we computed the power spectrum of the re-mapped output signal, which showed that the frequency of the re-mapped output signal was $300 \mathrm{~Hz}$. Moreover, we found that the PS of the re-mapped output and the PS of the original input signal were highly correlated $(r=0.978)$ (Fig. 4F). A correlation value of less than 1 implies that the algorithm concedes some spectral leakage during the mapping. However, because of the high correlation between the original and re-mapped signals, the leakage can be considered negligible.

\subsection{Experiment 2: Validation with frequency sweep sinusoid}

A frequency sweep sinusoidal input signal was generated with a sampling frequency of $16 \mathrm{kHz}$. The 
frequency o the signal input changed logarithmically from $21 \mathrm{~Hz}$ to $480 \mathrm{~Hz}$ in 1 a second period (Fig. 5A). The power spectrum of this input signal confirmed that the start and end frequencies of the signal were $21 \mathrm{~Hz}$ and $480 \mathrm{~Hz}$, respectively (Fig. 5B). The algorithm took this input signal and the mapping function (Eqn. 5a), and produced an output, which was also a frequency-sweep sinusoid, but with different start and end frequencies (Fig. 5C). Theoretically, according to the mapping function (Eqn. 5a), the start and end frequencies should be equal to $14 \mathrm{~Hz}$ and $320 \mathrm{~Hz}$, respectively. The power spectrum of the mapped output signal confirmed that the start and end frequencies were $14 \mathrm{~Hz}$ and $320 \mathrm{~Hz}$, respectively (Fig. 5D). Now, to investigate the spectral leakage, the mapped output signal and the re-mapping function (Eqn. 5b) were used as the inputs to the algorithm in a similar technique explained in experiment 1 . The algorithm was able to produce the original input signal $(\mathrm{r}=0.998)$ (Fig. 5E). Also, the two power spectra (Fig. 5B and $5 \mathrm{~F})$ were also highly correlated $(\mathrm{r}=0.996)$.

\subsection{Experiment 3: Validation with natural signals}

Seven natural signals were used to validate the algorithm. The signals were available in Matlab2018b. A description of the signals is listed in Table 2.

One of the seven natural signals was a train whistles signal (Fig 6A). This signal is more complicated than a pure tone or a frequency sweep sinusoid. It contains multiple frequencies (unlike pure tones) and its frequency at a given time can be lower or higher (unlike frequency sweeps) than the frequency of at the previous time point. Indeed, the power distribution over the frequency and time shows that the train whistles signal comprised several major frequency bands over the time (Fig. 6B). The presence of the multiple frequencies in the signal can also be verified by power spectrum, which shows that the power of the signal was concentrated at certain frequencies as indicated by the larger peaks on the power spectrum (Fig. 6C).

The train whistles signal along with the mapping function (Eqn. 5a) was given to the algorithm, which 
produced a mapped output signal (Fig. 6D). The overall envelope of the mapped output signal remained intact but the frequencies were changed as expected. The power of the mapped output signal essentially shifted to lower frequency bands as shown in its power distribution over frequency and time (Fig. 6E). Indeed, the frequencies of the original signal were reduced by a factor of $3 / 2$ (Eqn. 5a), which is more obvious in the frequency spectrum of the mapped output signal (Fig. 6F). Now, to investigate the spectral leakage, the mapped output signal and the re-mapping function (Eqn. 5b) were used as the inputs to the algorithm in a similar technique explained in experiment 1 . The algorithm was able to produce the original input signal $(\mathrm{r}=0.99)$ (Fig. 6G). Moreover, the distribution of power over the frequency and time was shifted back to the higher frequency bands (Fig. 6H). More precisely, the frequencies were increased by a factor of 3/2 (Eqn. 5b), which was more obvious in the power spectrum (Fig. 6I). The two power spectra (Fig. 6C and 6I) were also highly correlated $(\mathrm{r}=0.851)$.

Similar analyses were performed with the six remaining example natural signals (chirping of a bird; Hallelujah chorus from Handel's Messiah; fall and splat sound; sound of laughing of humans; male voice speaking; and rock with vocals, drums and guitar). We found that frequency spectra of the original input signal and the output signal obtained from the re-mapping process were highly correlated (Fig. 7A-F). High correlation values in all these examples with a wide variety of natural signals demonstrate that the algorithm can map the spectrum from one to another with a nominal leakage.

\section{Discussion}

This paper presents an algorithm to generate a modified and desired signal by changing the spectral contents of an input signal. The algorithm takes two inputs: (1) A time-domain signal that needs to be mapped; and (2) A frequency mapping function, which is used to map the input signal to a desired output signal. A 2-step process is used to generate the output signal from an input signal. The analysis step extracts the envelope and the instantaneous phases of the input signal. The instantaneous phases are then converted into the instantaneous frequencies, which are mapped according to the frequency mapping 
function. The mapped frequencies are then converted back to new phases, i.e., mapped phases. The synthesis step combines the extracted envelope and the mapped phases to construct the output signal. This paper provides the details of these steps with proper mathematical and conceptual explanations.

The performance of the algorithm was tested and validated by performing a series of experiments. In the first experiment, we used a pure tone sinusoidal signal along with a linear mapping function as the inputs to the algorithm and computed the mapped output. Inversely, the mapped output and the inverse linear function were used as the inputs to the model and reconstructed or estimated the original input signal. We showed that the original input and the estimated input signals were identical, which implies a negligible spectral loss in the mapping processes. Similarly, in the second experiment, we used a slightly more complicated input signal, a frequency sweep sinusoid, and showed that the original input and the estimated input signals were highly correlated. Finally, in the third experiment, we used seven natural signals with various level of complexities. The signals are available in Matlab2018b as train whistles, chirping of a bird, Hallelujah chorus from Handel's Messiah, fall and splat sound, sound of laughing of humans, male voice speaking, and rock with vocals, drums and guitar. For each of the original input signals and their corresponding estimated input signals via the inverse mapping process were highly correlated. The results of these experiments collectively demonstrate that the proposed algorithm concedes a negligible spectral leakage during the mapping of the input signals to the desired output signals.

The proposed algorithm can faithfully map the frequencies of a wide variety of natural signals with various levels of complexities, i.e., from pure tones to human voice with instruments. However, the algorithm also encounters a number of limitations. First, the algorithm relies on the estimation of instantaneous frequencies. So, without a good estimation of the instantaneous frequency, the algorithm will perform poorly. The method of estimating frequencies used in this paper has widely been studied, but a better method will increase the performance of the algorithm. Second, spectral leakage is higher for 
complex signals as compared to simple signals like pure tones. Again, the estimation of instantaneous frequencies of the complex signals is less accurate. This error in estimation may be accounted for as the spectral leakage. Third, the computational complexity of the algorithm has not been assessed in this paper, and is left for the future development. In general, the algorithm performs pretty fast as it uses Fast Fourier Transform (FFT) to compute Hilbert transform in the analysis step. For instance, each example in this paper took a fraction of seconds to perform mapping and re-mapping using Matlab 2018b in a MacOSX (1.4 GHz, Intel Core i5, 4GB RAM). Larger signals may be partitioned in time and each partition can be used as a separate input to make the computation faster, which is out of scope of this paper.

Some potential fields of applications of the signal mapping algorithms may include but not limited to music industries, artificial intelligence, and neuroscience. Particularly, this algorithm can be applied to transform perceptual signals from one modality to the other, e.g., from audio frequency to tactile and vice versa (Rahman et al., 2017; Convento et al., 2018; Rahman and Yau, 2019; Rahman et al., 2019; Rahman et al., 2020). These applications will potentially provide us new insights to understand perceptual links between different sensory systems.

\section{Authors Contributions}

M.S.R. developed the algorithms, designed and performed experiments and analyses, and wrote the manuscript. 


\section{Data Availability}

Data and codes are available upon request to the author.

\section{Competing Interests}

Author declares no conflict of interests.

\section{References}

Alluri V and Toiviainen P. Exploring perceptual and acoustic correlates of polyphonic timbre. Music Perception: An Interdisciplinary Journal, 27(3):223-242, 2010. DOI: 10.1525/mp.2010.27.3.223

Bonnel J, Caporale S and Thode A. Waveguide mode amplitude estimation using warping and phase compensation. Journal of the Acoustic Society of America, 141(3):2243, 2017. doi: $10.1121 / 1.4979057$

Chang SA, Won JH, Kim H, Oh SH, Tyler RS and Cho CH. Frequency-limiting effects on speech and environmental sound identification for cochlear implant and normal hearing listeners. Journal of Audiology and Otology, 22(1):28-38, 2017. doi: 10.7874/jao.2017.00178

Chemin B, Huang G, Mulders D and Mouraux A. EEG time-warping to study non-strictly-periodic EEG signals related to the production of rhythmic movements. J Neurosci Methods, 308:106-115, 2018. doi: 10.1016/j.jneumeth.2018.07.016

Convento S, Rahman MS and Yau JM. Selective attention gates the interactive crossmodal coupling between perceptual systems. Current Biology, 28: 746-752, 2018. doi: 10.1016/j.cub.2018.01.021

[1]Deutsch D. The psychology of music. $3^{\text {rd }}$ Edition, 2013. Paperback ISBN: 9780123814609

eBook ISBN: 9780123814616

Dolson M. The phase vocoder: A tutorial. Computer Music Journal, 10(4):14-27, 1986. DOI: $10.2307 / 3680093$

Flanagan JL and Golden RM. Phase vocoder. Bell System Technical Journal, 45:1493-1509, 1966. DOI: 10.1002/j.1538-7305.1966.tb01706.x 
Goupell MJ, Laback B, Majdak P and Baumgartner WDB. Effects of upper-frequency boundary and spectral warping on speech intelligibility in electrical simulation. Journal of the Acoustic Society of America, 123(4):2295-2309, 2008. doi: 10.1121/1.2831738

Grey JM and Gordon JW. Perceptual effects of spectral modifications on musical timbers. Journal of the Acoustic Society of America, 63:1493-1500, 1978. DOI: 10.1121/1.381843

Grey JM and Moorer JA. Perceptual evaluations of synthesized musical instrument tones. Journal of the Acoustic Society of America, 62:454-462, 1977. DOI: 10.1121/1.381508

Han K, Wang Y, Wang D, Woods WS, Merks I and Zhang T. Learning spectral mapping for speech dereverberation and denoising. IEEE/ACM Transactions on Audio, Speech, and Language Processing, 23: 982-992, 2015. DOI: 10.1109/TASLP.2015.2416653

Krumhansl CL and Iverson P. Perceptual interactions between musical pitch and timbre. Journal of Experimental Psychology: Human Perception and Performance, 18(3):739-751, 1992. DOI: $10.1037 / 0096-1523.18 .3 .739$

Laroche J and Dolson M. New phase vocoder technique for pitch-shifting, harmonizing and other exotic effects. Proc. IEEE Workshop on Applied Signal Processing and Audio and Acoustics, New Paltz, NY, 1999. DOI: 10.1109/ASPAA.1999.810857

Lee L and Rose R. A frequency warping approach to speaker normalization. IEEE Transactions on Speech and Audio Processing, 6(1):49-60, 1998. DOI: 10.1109/89.650310

Meszlényi RJ, Hermann P, Buza K, Gál V and Vidnyánszky Z. Resting state fMRI functional connectivity analysis using dynamic time warping. Frontiers of Neuroscience, 11:75, 2017. doi: $10.3389 /$ fnins. 2017.00075

Moorer JA. The use of the phase vocoder in computer music applications. Journal of the Audio Engineering Society, 24(9):717-727, 1978.

Niu $\mathrm{H}$, Zhang $\mathrm{R}$ and $\mathrm{Li} \mathrm{Z}$. Theoretical analysis of warping operators for non-ideal shallow water waveguides. Journal of the Acoustic Society of America, 136(1):53-65, 2014. doi: 10.1121/1.4883370 
Noda H. Frequency-warped spectral distance measures for speaker verification in noise. ICASSP, 1:576-579, 1988. DOI: 10.1109/ICASSP.1988.196650

Puckette M. Phase-locked vocoder. Proc. IEEE ASSP Conference on Applied Signal Processing, Audio and Acoustics, New Paltz, NY, 1995. DOI: 10.1109/ASPAA.1995.482995

Puckette MS and Brown JC. Accuracy of frequency estimates using the phase vocoder. IEEE Transactions on Speech and Audio Processing, 6(2):166-176, 1998. DOI: 10.1109/89.661475

Qiang LZ, Wen DW, Qing L and Telfer D. Texture image segmentation: a local spectral mapping approach. Proceedings of 3rd IEEE International Conference on Image Processing. 1996. DOI: 10.1109/ICIP.1996.560383

Rahman MS. Spectral remapping of natural signals. arXiv preprint, arXiv:1912.10371, 2019.

Rahman MS. Computations, optimization and tuning of deep feedforward neural networks. bioRxiv 889311 preprint, 2019. doi: https://doi.org/10.1101/2019.12.27.889311

Rahman MS. Computational design of cardiac activity. International Journal of Medicine and Medical Sciences, 3(10): 321-330, 2011

Rahman MS, Barnes KA, Crommett LE, Tommerdahl and Yau JM. Auditory and tactile frequency representations are co-embedded in modality-defined cortical sensory systems. NeuroImage, 215: 116837, 2020. https://doi.org/10.1016/j.neuroimage.2020.116837

Rahman MS, Barnes KA, Crommett LE, Tommerdahl and Yau JM. Multimodal frequency representations are embedded in modality-defined cortical systems. bioRxiv preprint, 2019. doi: https://doi.org/10.1101/628305

Rahman MS and Haque MA. Mathematical modeling of blood flow. IEEE International Conference on Informatics, Electronics and Vision, pp: 672-676, 2012. DOI: 10.1109/ICIEV.2012.6317446

Rahman MS, Patel AM and Yau JM. Probabilistic inference of multi-finger touch. Conference on Cognitive and Computational Neuroscience, pp:1-2, 2017.

Rahman MS and Yau JM. Somatosensory interactions reveal feature-dependent computations. Journal of Neurophysiology, 122: 5-21, 2019. doi: 10.1152/jn.00168.2019 
Samson S, Zatorre RJ and Ramsay JO. Multidimensional scaling of synthetic musical timber: Perception of spectral and temporal characteristics. Canadian Journal of Experimental Psychology, 51(4):307-315, 1997. DOI: $10.1037 / 1196-1961.51 .4 .307$

Shoaib MSR, Asaduzzaman M and Haque MA. Mathematical modeling of the heart. IEEE International Conference on Electrical and Computer Engineering. 6: 626-629, 2010. DOI: 10.1109/ICELCE.2010.5700771

Wang L, Arends JB, Long X, Wu Y and Cluitmans PJ. Seizure detection using dynamic warping for patients with intellectual disability. IEEE EMBS, 1010-1013, 2016. doi: 10.1109/EMBC.2016.7590873

von Schroeter T. Frequency warping with arbitrary allpass map. IEEE Signal Processing Letters, 6(5):116-118, 1999. DOI: 10.1109/97.755432

Vos J and Rasch R. The perceptual onset of musical tones. Perception \& Psychophysics, 29(4):323-335, 1981. doi.org/10.1007/978-1-4684-8917-0_16

Wabnik S, Schuller G, Kramer U and Hirschfeld J. Frequency warping in low delay audio coding. ICASSP, 2005. DOI: 10.1109/ICASSP.2005.1415676 


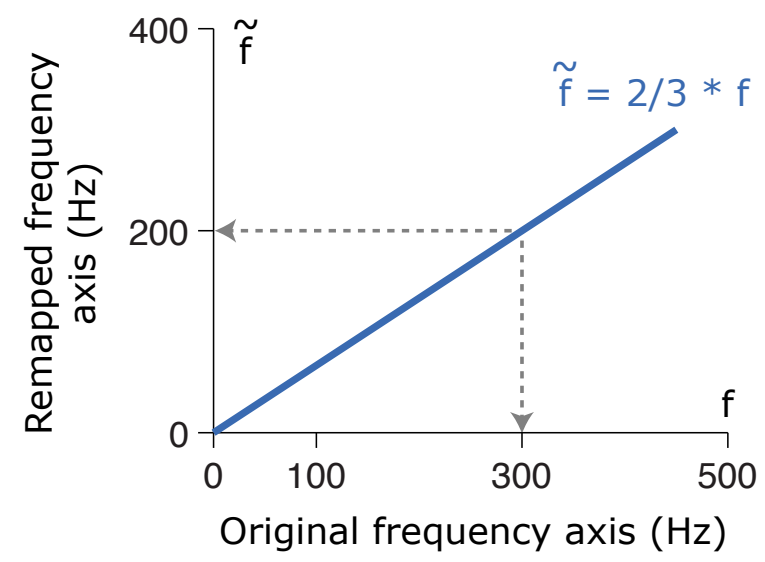

Figure 1. Frequency remapping explained with a warping or mapping function, $\tilde{\mathrm{f}}=2 / 3 * \mathrm{f}$, where $\mathrm{f}$ and $\tilde{\mathrm{f}}$ are the frequencies of the original and mapped signals, respectively. So, a frequency of $300 \mathrm{~Hz}$ in original axis will be projected to $200 \mathrm{~Hz}$ in mapped axis, and vice versa. 


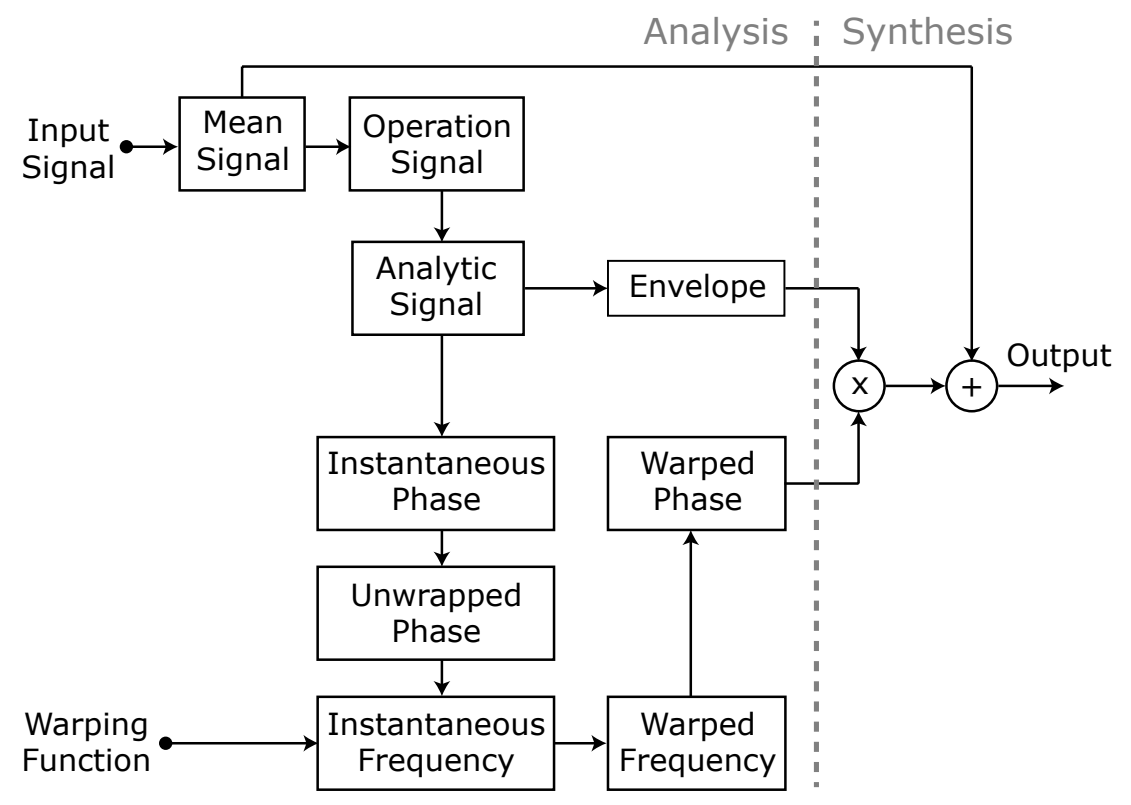

Figure 2. Block diagram representation of the frequency warping algorithm. The input signal is used to compute the mean signal and the operation signal. The analytic signal is computed using the Hilbert transform on the operation signal. The analytic signal provides information about the envelope and instantaneous phase of the signal. The instantaneous phase is unwrapped to obtain instantaneous frequency. A warping function is applied to transform instantaneous frequency into warped frequency, which is converted into warped phase. The envelope and the warped phases are multiplied (element-wise product), which is then added with the mean signal to obtain the warped output signal. The vertical dashed line indicates the separation between the operations during analysis and synthesis steps. 

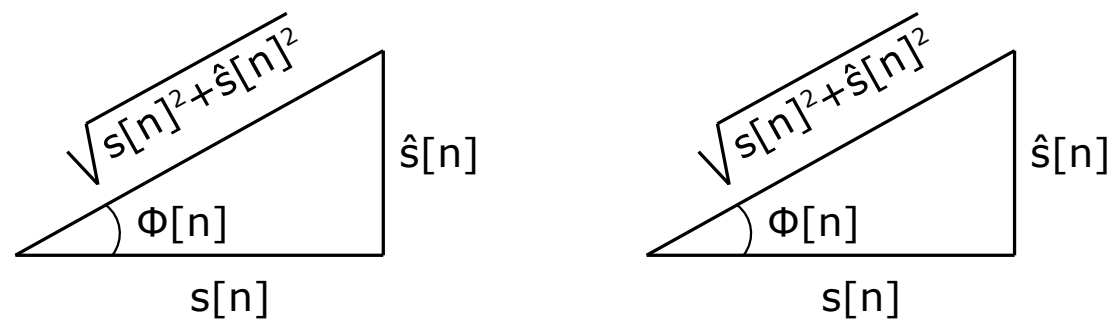

Figure 3. Trigonometric identities. A) For the input signal. To make this triangular representation analogous to equation (6), base and height must be $s[n]$ and $\hat{s}[n]$, respectively. Accordingly, the hypotenuse is $\sqrt{s[n]^{2}+\hat{s}[n]^{2}}$, which is equal to the envelope e[n]. Thus, $\arctan \left(\frac{\hat{s}[n]}{s[n]}\right)=\phi[n]=$ $\arccos \left(\frac{\mathrm{s}[\mathrm{n}]}{\mathrm{e}[\mathrm{n}]}\right)$. B) For the desired output signal. 

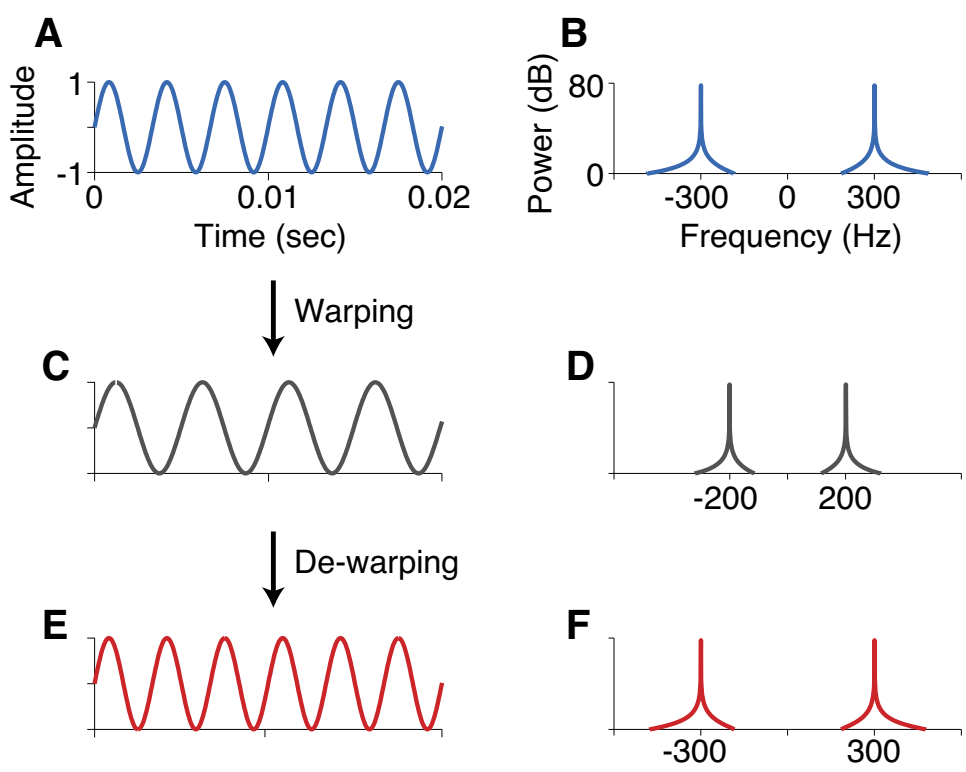

Figure 4. Validation of frequency warping algorithm with a pure tone sinusoid. A) Pure tone sinusoidal input signal with a frequency of $300 \mathrm{~Hz}$. Original signal was 1 second long; a shorter version is shown for better visualization. B) Power spectrum density (PSD) of the input signal. C) Warped signal obtained after applying frequency warping function on the input signal. D) PSD of warped signal. E) De-warped signal obtained after applying inverse warping function on warped signal. F) PSD of de-warped signal. For a lossless algorithm, de-warped signal presented in E must be same to the original input presented in A, therefore, PSD presented in F must be same to that in B. 
A
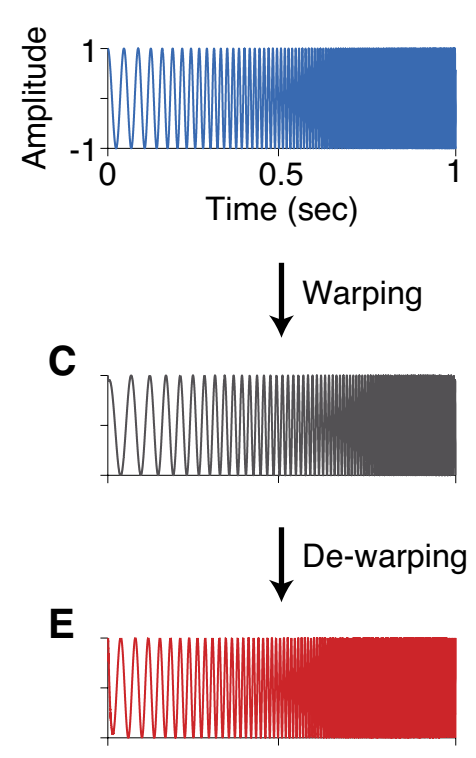

B

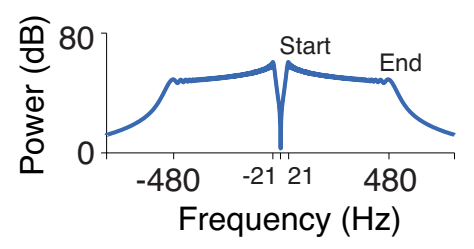

D

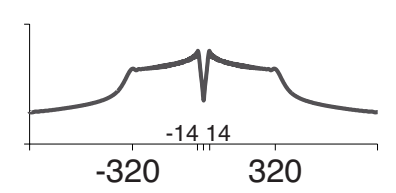

$\mathbf{F}$

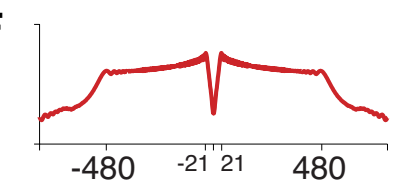

Figure 5. Validation of frequency warping algorithm with a frequency sweep sinusoid. A) Frequency sweep sinusoidal input signal, whose frequency increases logarithmically from $21 \mathrm{~Hz}$ to $480 \mathrm{~Hz}$ in 1 second. B) PSD of the input signal showing the start and end frequencies. C) Warped signal obtained after applying frequency warping function on the input signal. D) PSD of warped signal. E) De-warped signal obtained after applying inverse warping function on warped signal. F) PSD of de-warped signal. For a lossless algorithm, de-warped signal presented in E must be same to the original input signal presented in A, therefore, PSD presented in F must be same to that in B. 

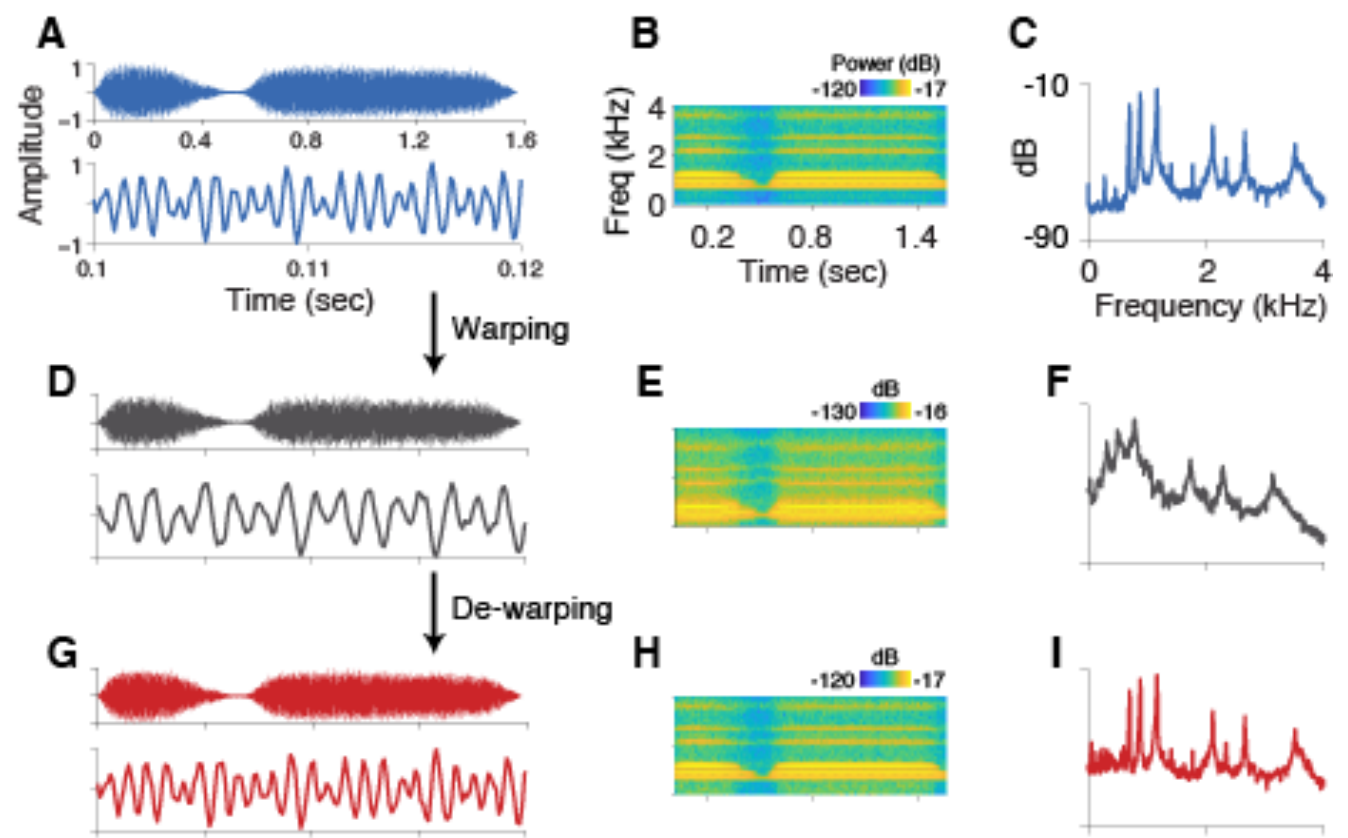

Figure 6. Validation of the algorithm with more common natural signal. A) Top: Original input signal of train whistles (train.mat, Fs $=8192 \mathrm{~Hz}, 1.57$ seconds). Bottom: Input signal zoomed-in between 0.1 and 0.12 seconds. B) Time-frequency plot of power distribution of input signal. C) PSD of the input signal. D) Warped signal (top: entire duration, bottom: zoomed-in version). E) Time-frequency plot of power distribution of warped signal. F) PSD of the warped signal. G) De-warped signal (top: entire duration, bottom: zoomed-in version). H) Time-frequency plot of power distribution of de-warped signal. I) PSD of the de-warped signal. 

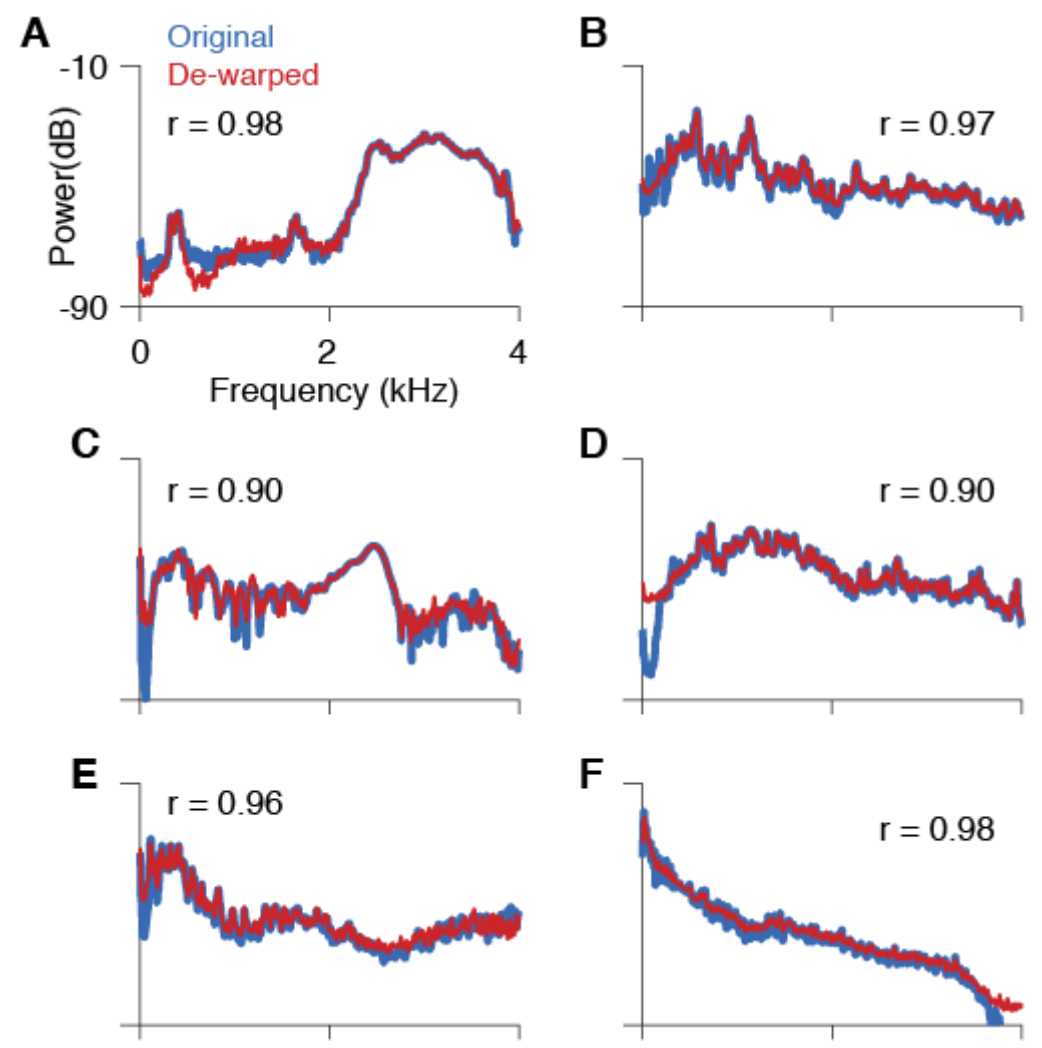

Figure 7. Validation with different natural signals. Power spectrum of: A) Chirping of a bird. B) Hallelujah chorus from Handel's Messiah. C) Fall and splat sound. D) Sound of laughing of humans. E) Male voice speaking. F) Rock with vocals, drums and guitar (used Fs $=8000,53.78$ seconds to keep consistent with sampling frequencies of the other signals in this figure. Conclusions are not changed when using Fs $=48000 \mathrm{~Hz}$ ). 
Table 1. Summary of the algorithm

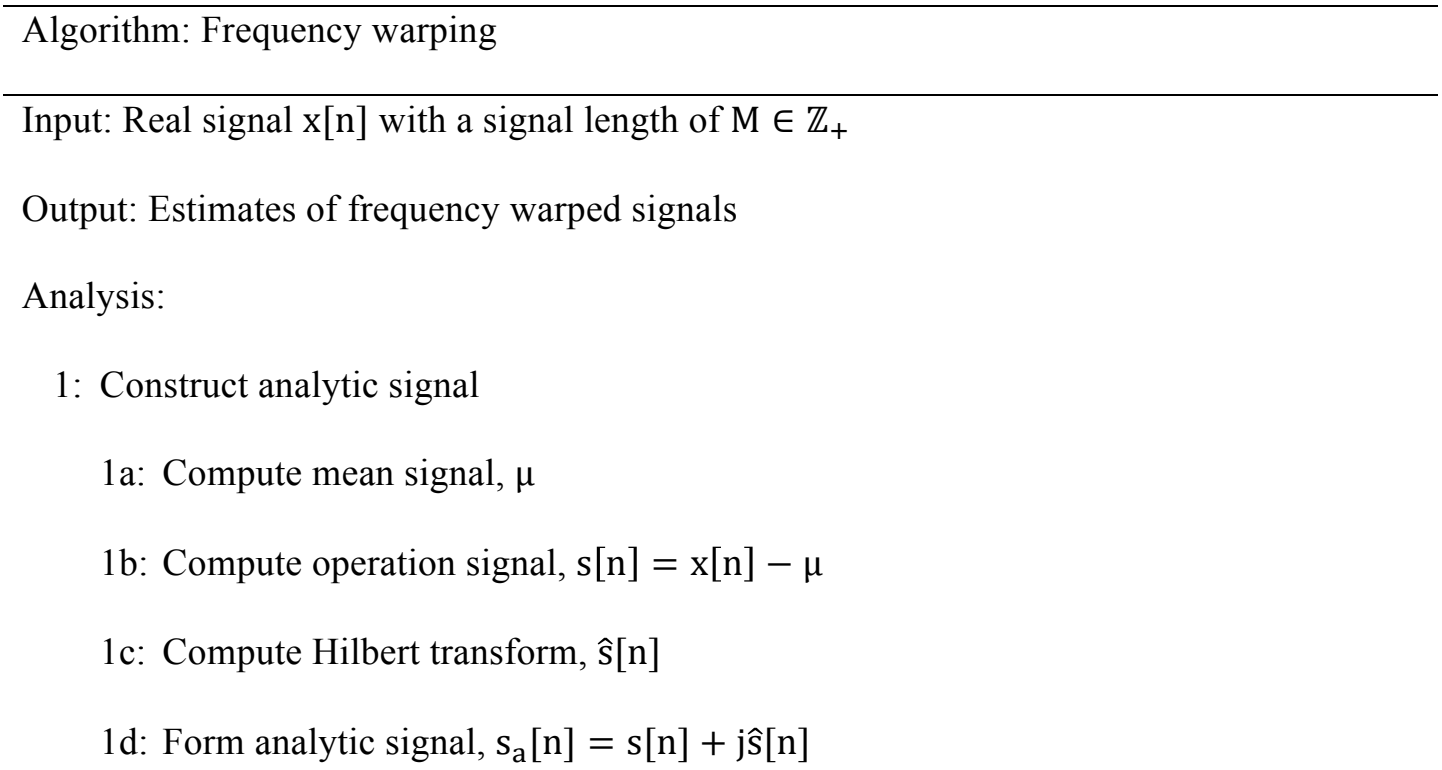

2: Extract properties of the analytic signal

2a: Extract envelope, $e[n]=\left|s_{a}[n]\right|$

2b: Extract instantaneous phase, $\phi[n]=\Varangle s_{a}[n]$

3: Warp frequency and phase

3a: Unwrap phase, $\theta[n]=\mathfrak{U}(\phi[n])$

3b: Obtain instantaneous frequency, $\mathrm{f}[\mathrm{n}]$

3c: Apply frequency warping function and obtain warped frequency, $\tilde{\mathrm{f}}[\mathrm{n}]$

3d: Convert $\tilde{\mathrm{f}}[\mathrm{n}]$ into warped phase, $\widetilde{\phi}[\mathrm{n}]$

Synthesis:

4: Reconstruct warped output signal

4a: Perform dot product between the envelope and the cosine of wrapped phases

4b: Add the product to the mean signal and obtain the warped output signal, $\tilde{\mathrm{x}}[\mathrm{n}]$ 
Table 2. Natural signals used for validation

\begin{tabular}{|c|c|c|}
\hline Signals (name and format available in Matlab2018b) & Fs $(\mathrm{Hz})$ & Duration (sec) \\
\hline Whistles of a train (train.mat) & 8192 & 1.57 \\
\hline Chirping of a bird (chirp.mat) & 8192 & 1.5 \\
\hline Hallelujah chorus (handel.mat) & 8192 & 8.92 \\
\hline Fall and splat sound (splat.mat) & 8192 & 1.22 \\
\hline Sound of laughing (laughter.mat) & 8192 & 6.42 \\
\hline 6. Male voice speaking (speech_dft_8kHz.wav) & 8000 & 4.99 \\
\hline 7. $\quad$ Rock with vocals, drums and guitar (audio48kHz.wav) & 48000 & 8.96 \\
\hline
\end{tabular}

